\title{
Derivation and Application of Sensitivities to Assess Transient Voltage Sags caused by Rotor Swings th
}

\author{
Tilman Weckesser ${ }^{\mathrm{a}, *}$, Hjörtur Jóhannsson ${ }^{\mathrm{a}}$, Jacob Østergaard ${ }^{\mathrm{a}}$, \\ Thierry Van Cutsem ${ }^{\mathrm{b}}$ \\ ${ }^{a}$ Center for Electric Power and Energy (CEE) at the Technical University of Denmark \\ (DTU), Kgs. Lyngby, Denmark \\ ${ }^{b}$ Fund for Scientific Research (FNRS) at the University of Liège, Belgium
}

\begin{abstract}
The paper introduces an approach to investigate voltage sags, which are caused by large generator rotor swings following a transient disturbance. Therefore, the method exploits sensitivities derived from the algebraic network equations. These provide information on the impact of a generator on the voltage magnitude at a load bus and the effect of load variation on the generator's power injection. It is shown that these sensitivities give valuable information to identify critical generator-load pairs and locations for applying preventive control measures.
\end{abstract}

Keywords: power system stability, sensitivity to rotor swings, transient stability, voltage sag

\section{Introduction}

In literature on power quality, voltage sags/dips is a topic vastly addressed [2]. While the primary cause is the occurrence of a fault, a less pointed out reason for voltage sags is rotor angle swing, more precisely angular separation of generators, resulting from a fault. From a practical viewpoint, a scenario may be assessed transiently stable considering that

\footnotetext{
${ }^{2}$ An earlier version of the paper [1] was published as part of the proceedings of the Power Systems Computation Conference, August 18-22, 2014, Wroclaw, Poland.

Research supported by the Danish Council for Strategic Research (DSF).

${ }^{*}$ Corresponding author

Email addresses: jtgw@elektro.dtu.dk (Tilman Weckesser), hjjo@elektro.dtu.dk (Hjörtur Jóhannsson), joe@elektro.dtu.dk (Jacob Østergaard),

t.vancutsem@ulg.ac.be (Thierry Van Cutsem)

To appear in International Journal of Electrical Power and Energy Systems2015
} 
generators remain in synchronism, while voltage sags due to the relative rotor angle displacement already result in transiently low voltages for which the system response should be considered unacceptable.

The prediction of this type of voltage sag using the Transient Energy Function was described in the early reference [3]. In [4] sensitivities relative to voltage dip were derived using this method as well. The sensitivities relate the voltage sag depths to certain parameters such as terminal voltages and power generation. The authors of [5] address the transient voltage dip acceptability problem using a two-dimensional table of critical voltage level and critical voltage dip duration. Moreover, the issue of transient voltage stability of dynamic loads such as induction machines is analysed. In the more recent reference [6] the authors use sensitivities to carry out contingency filtering and ranking with respect to voltage dips. Furthermore, the assessment addresses power quality issues and short-term voltage stability. In [7, the authors present a survey of current practices for transient voltage sag criteria related to power system stability.

The present paper also investigates voltage sags with focus on power system stability rather than power quality. Transient voltage sags are identified using time-domain simulation. Then, sensitivities are derived which provide information on tight couplings between relative change of rotor angles and load voltage magnitudes. These sensitivities are easier to compute than those considered in the above references. The sensitivities can be used, for instance, to identify the contribution of each generator to a drop in voltage magnitude experienced at a particular load bus. A voltage depression at a load bus can trigger consecutive events such as load tripping. Therefore, a second sensitivity is derived, which assesses the impact of a change of load power on generator active powers.

This paper is organized as follows. In Section 2 the power system model used for the discussion of voltage sags and the computation of sensitivities is described. Moreover, a brief discussion of the mechanism causing these transient voltage sags is presented. The voltage sensitivities are derived in Section 3. This is followed by the presentation of the corresponding results in Section 4. The derivation of the second sensitivities, addressing the effect of variation of load on generator power, can be found in Section 5 and the corresponding results are shown in Section 6. Finally, in Section 7 concluding remarks are offered. 


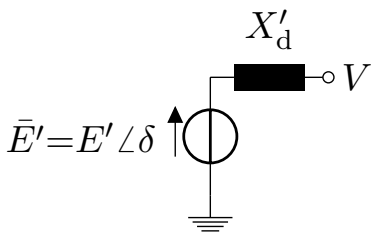

(a) Thevenin Equivalent

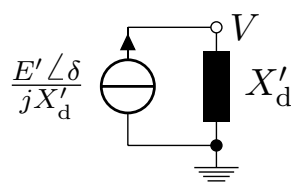

(b) Norton Equivalent

Figure 1: Thévenin and Norton equivalents of generator

\section{Voltage sags caused by rotor angle swings}

\subsection{Modelling for discussion of the mechanism causing the voltage sags and for sensitivity analysis}

The model used for synchronous generator is the so-called "classical" transient stability model [8]. Each generator is modeled by an e.m.f. $\bar{E}^{\prime}$ of constant magnitude behind the transient reactance $X_{\mathrm{d}}^{\prime}$ (see Fig. 1(a), the mechanical power input is assumed constant, and loads are converted to constant shunt admittances. The simple generator model is valid in the first second after fault clearance and the justification for using it is twofold. First, this model is used for sensitivity analysis and, as indicated in the Introduction, this analysis is aimed at complementing a detailed time-domain simulation in which much more refined models can be used. Second, the "classical" model is not used with constant e.m.f. throughout the whole simulation: instead, the e.m.f. is adjusted so that the "classical" model fits specific operating points where the sensitivity analysis is carried out.

For convenience each generator is represented by its Norton equivalent (see Fig. $1(\mathrm{~b})$, i.e. a current source $\bar{E}^{\prime} /\left(j X_{\mathrm{d}}^{\prime}\right)$ in parallel with the admittance $1 /\left(j X_{\mathrm{d}}^{\prime}\right)$. Based on these assumptions, the following well-known linear algebraic equations can be used:

$$
\overline{\boldsymbol{I}}=\boldsymbol{Y} \overline{\boldsymbol{V}}
$$

where $\overline{\boldsymbol{I}}$ is the vector of complex currents injected at the generator buses (stemming from the Norton equivalents), $\overline{\boldsymbol{V}}$ is the vector of complex bus voltages, and $\boldsymbol{Y}$ is the "augmented" bus admittance matrix obtained by adding the contribution of generators and loads to the matrix relative to the network.

A system with $n$ buses and $m$ machines is considered $(n>m)$. It is assumed, without loss of generality, that the buses where machines are connected are numbered from $n-m+1$ to $n$. Hence, Eq. (1) can be detailed 


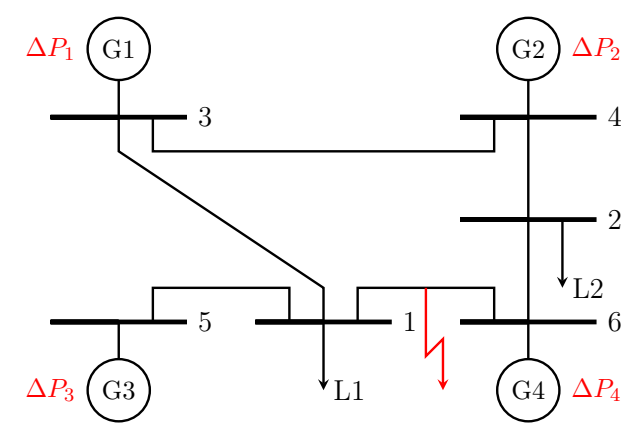

Figure 2: One-line diagram of a simple example test system with 6 buses. A three-phase short-circuit on the transmission line connecting buses 1 and 6 , which is indicated by the red zigzag arrow, causes a change $\Delta P_{i}$ in the power injection of each generator $\mathrm{G} i$.

as:

$$
\left[\begin{array}{c}
0 \\
\vdots \\
0 \\
\bar{E}_{1}^{\prime} /\left(j X_{\mathrm{d}, 1}^{\prime}\right) \\
\vdots \\
\bar{E}_{m}^{\prime} /\left(j X_{\mathrm{d}, m}^{\prime}\right)
\end{array}\right]=\boldsymbol{Y}\left[\begin{array}{c}
\bar{V}_{1} \\
\vdots \\
\bar{V}_{n-m} \\
\bar{V}_{n-m+1} \\
\vdots \\
\bar{V}_{n}
\end{array}\right]
$$

where the zero sub-vector has dimension $n-m$ and $\bar{V}_{i}$ is the complex voltage at the $i$-th bus.

\subsection{Voltage sag mechanism}

The mechanism behind voltage sags caused by rotor swings can be discussed and illustrated graphically under consideration of a simple example system such as the one shown in Fig. 2.

As mentioned in the previous section the generators are represented by the "classical" transient stability model. The angle of the e.m.f. can then be used to represent the rotor angle of the generator [8]. Moreover, in this example the generators' mechanical powers are assumed constant and the loads are modelled as constant impedances for sake of simplicity.

With these and the aforementioned assumptions, Eq. (2) reveals that the complex voltage at a bus is the sum of contributions of the generators. The contribution of a generator is determined by its e.m.f., which is scaled and rotated corresponding to the entry in the inverse of the admittance matrix divided by the respective transient reactance. 


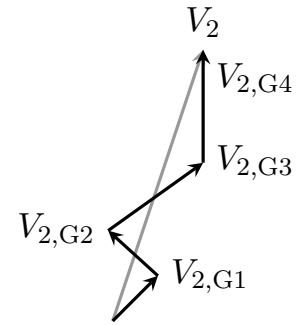

(a) Bus voltage at initial state

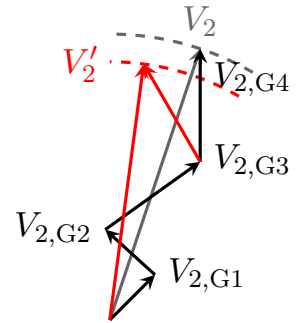

(b) Effect of rotor angle increase of generator G4

Figure 3: Example of complex voltage at Bus 2 as sum of generator contributions

Figure 3(a) shows the complex voltage at Bus 2 (from the simple example system) and the contributions of the individual generators, which add up to the voltage measured at the respective bus.

The effect of an increase in rotor angle, e.g. due to a transient disturbance, on a particular bus voltage can be assessed under consideration of the linear algebraic equation Eq. (2).

In the example shown in Fig. 2, a short-circuit occurs on the transmission line connecting Bus 4 and 5. The fault alters the admittance matrix and leads to a change of the electric power injection of the generators, which causes a relative acceleration or deceleration of the generator rotors. In the example, it is assumed for clarity that the fault only affects generator G4 resulting in its acceleration and an advancing of its rotor angle relative to the remaining generators.

The effect on the voltage at Bus 6 is shown in Fig. 3(b) from which it can be observed that the relative increase in rotor angle depresses the voltage magnitude at the bus. This observation gave the impulse for the investigation of transient voltage sags using sensitivities.

\section{Derivation of Load Voltage Sensitivities}

\subsection{Load voltage sensitivities}

In this section, sensitivities are derived to identify the load buses whose voltage magnitudes are strongly affected by changes of the rotor angle of particular generators. They are referred to as "load voltage sensitivities". The latter will be used to determine the contribution of individual generators to an observed voltage sag at a load bus. 
The effect of a small rotor angle change is assessed through modification of Eq. (2). To this purpose, the e.m.f. phasor of the $k$-th generator, $\bar{E}_{k}^{\prime}$, is slightly rotated, while the other generators remain unchanged. This corresponds to a small change of the rotor angle, when generators are represented by the "classical" model. The new bus voltages $\overline{\boldsymbol{V}}^{\dagger}$ that result from a small increase $\epsilon$ in the phase angle of $\bar{E}_{k}^{\prime}$ are easily computed by solving the modified linear system:

$$
\left[\begin{array}{c}
0 \\
\vdots \\
0 \\
\bar{E}_{1}^{\prime} /\left(j X_{\mathrm{d}, 1}^{\prime}\right) \\
\vdots \\
\bar{E}_{k}^{\prime} e^{j \epsilon \pi / 180 /\left(j X_{\mathrm{d}, k}^{\prime}\right)} \\
\vdots \\
\bar{E}_{m}^{\prime} /\left(j X_{\mathrm{d}, m}^{\prime}\right)
\end{array}\right]=\boldsymbol{Y}\left[\begin{array}{c}
\bar{V}_{1}^{\dagger} \\
\vdots \\
\bar{V}_{n-m}^{\dagger} \\
\bar{V}_{n-m+1}^{\dagger} \\
\vdots \\
\vdots \\
\vdots \\
\bar{V}_{n}^{\dagger}
\end{array}\right]
$$

In order to speed up computations and make the approach well suited for real-time application, the admittance matrix $\boldsymbol{Y}$ is $L U$-factorized once for all, and Eq. (3) is solved for each change $\epsilon$ in the left-hand-side vector.

The changes of magnitudes between the "new" and the reference bus voltages are calculated and normalized. The sensitivities of the various bus voltage magnitudes to the $i$-th generator rotor angle are computed as:

$$
\left(s_{\mathrm{V}, \mathrm{G} i}\right)_{\ell}=\frac{\left|\bar{V}_{\ell}^{\dagger}\right|-\left|\bar{V}_{\ell}\right|}{\pi \epsilon / 180} \quad \ell=1, \ldots, m-n
$$

where $\epsilon$ is expressed in degrees. The resulting sensitivity vector has the unit $[\mathrm{pu} / \mathrm{rad}]$ or $[\mathrm{V} / \mathrm{rad}]$. This vector can be determined for each generator in the system and the aggregation of the individual vectors gives a sensitivity matrix $\boldsymbol{S}_{\mathrm{V}, \mathrm{G}}$, where each column corresponds to a generator and each row to a load bus. This matrix allows identifying the generators having a dominant impact on a certain load bus voltage magnitude. The entries also indicate whether increasing a rotor angle will increase a voltage magnitude or will depress it.

\subsection{Contribution of individual generators to a voltage sag}

Once it has been found from time simulation that a significant voltage sag is experienced at some load bus, the voltage sensitivities presented in the 
previous sub-section can be utilized to identify the contribution of each individual generator. The procedure described hereafter was found to provide the most accurate results.

The time-domain simulation is carried out until the minimum voltage is reached at the load bus of interest. In order to ignore bus voltage variations caused by topological changes, the voltage magnitude reduction is considered between the time $t_{\mathrm{c}}$ of fault clearing and the instant $t_{\mathrm{Vmin}}$ where the minimum of voltage is reached. The excursions $\Delta \delta_{j}(j=1, \ldots, m)$ of rotor angles are considered over the time interval $\left[t_{\mathrm{c}} t_{\mathrm{Vmin}}\right]$. Furthermore, the load voltage sensitivities $\boldsymbol{S}_{\mathrm{V}, \mathrm{G}}$ are determined at time $t_{\mathrm{c}}^{+}$. Therefore, the bus voltages $V\left(t_{\mathrm{c}}^{+}\right)$and the power consumption of the loads just after the system has entered its post-fault configuration are used to update the admittance matrix and, subsequently, to compute the Norton equivalent of the generators.

Therewith, an estimate of the voltage magnitude drop at Bus $i$ can be calculated through linearisation, using the load voltage sensitivities, as follows:

$$
\Delta V_{i}=\sum_{j=1}^{m}\left[\boldsymbol{S}_{\mathrm{V}, \mathrm{G}}\right]_{i j} \Delta \delta_{j}=\sum_{j=1}^{m} \Delta V_{i, j}
$$

Finally, the share of the $k$-th generator in the total change of voltage magnitude can be determined as:

$$
f_{i, k}=\frac{\Delta V_{i, k}}{\left|\sum_{j=1}^{m} \Delta V_{i, j}\right|}
$$

Using this fraction and the voltage trajectory obtained from time-domain simulation, the actual share of the $k$-th generator in the voltage sag at the $i$-th bus can be computed as:

$$
\Delta V_{i, k}^{*}=f_{i, k}\left|V_{i}\left(t_{\mathrm{c}}^{+}\right)-V_{i}\left(t_{\mathrm{V} \min }\right)\right|
$$

where $V_{i}\left(t_{\mathrm{c}}^{+}\right)$is the voltage at Bus $i$ immediately after fault clearing and $V_{i}\left(t_{\mathrm{Vmin}}\right)$ its minimum value.

\section{Results for Load Voltage Sensitivities}

\subsection{Test system and example case}

\subsubsection{Test system}

The test system employed to validate the presented sensitivities is the well-known New England \& New York system described in [9]. It consists of 68 buses and 16 generators. A one-line diagram is shown in Fig. 4. The loads 
are modelled as constant impedances in the time-domain simulation. The generators are represented by a sixth order model. They all have a simple excitation and voltage regulation system, and a thermal turbine/governor model. Furthermore, all generators, but GEN-7 and GEN-14, are equipped with a power system stabilizer.

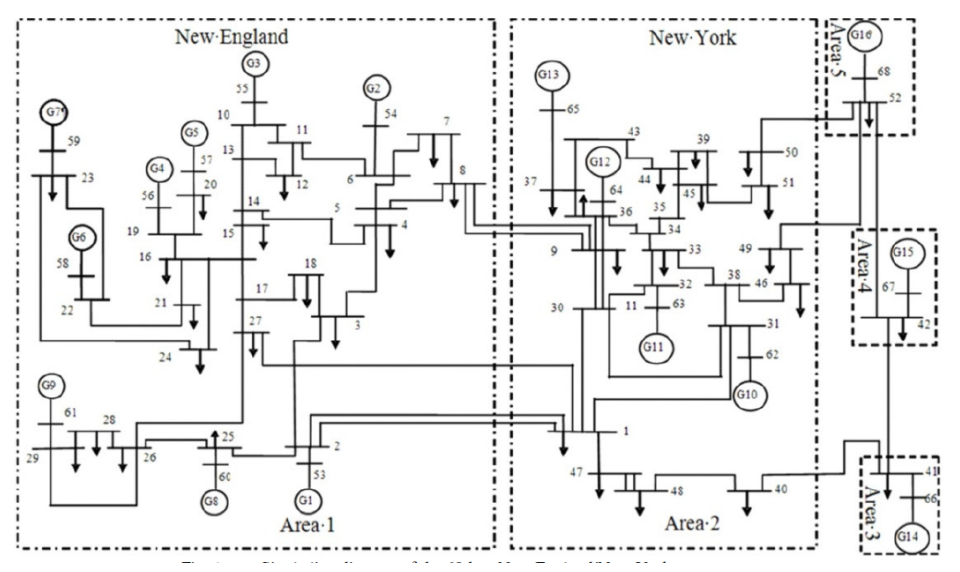

Figure 4: One-line diagram of the New England and New York system [10].

\subsubsection{Test scenario}

The test scenario was as well adopted from [9]. In this case, the considered contingency is a three-phase short-circuit on the transmission line connecting Buses 26 and 28. The short-circuit occurs one second after the simulation begins and is assumed to be very close to Bus 28 . It is cleared after $120 \mathrm{~ms}$ by opening the breakers at both ends of the faulted transmission line.

The disturbance causes some generators to accelerate relative to the others, which leads to a significant separation of the generator rotor angles. Figure 5 shows the evolution of the rotor angles of nine among the sixteen generators: the five generators with the largest increase of rotor angle (GEN-4, GEN-5, GEN-6, GEN-7 and GEN-9), the three generators with the largest decrease (GEN-14, GEN-15 and GEN-16) and one which is little affected (GEN-8). The graph shows that the generators are affected to different degrees by the disturbance; in particular, GEN-9 is experiencing a large rotor angle excursion. However, all generators remain in synchronism and reach a new stable equilibrium point. Consequently, the scenario is assessed to be stable. Figure 6 displays the time evolution of voltage magnitudes at a selection of load buses. The selection consists of the four 


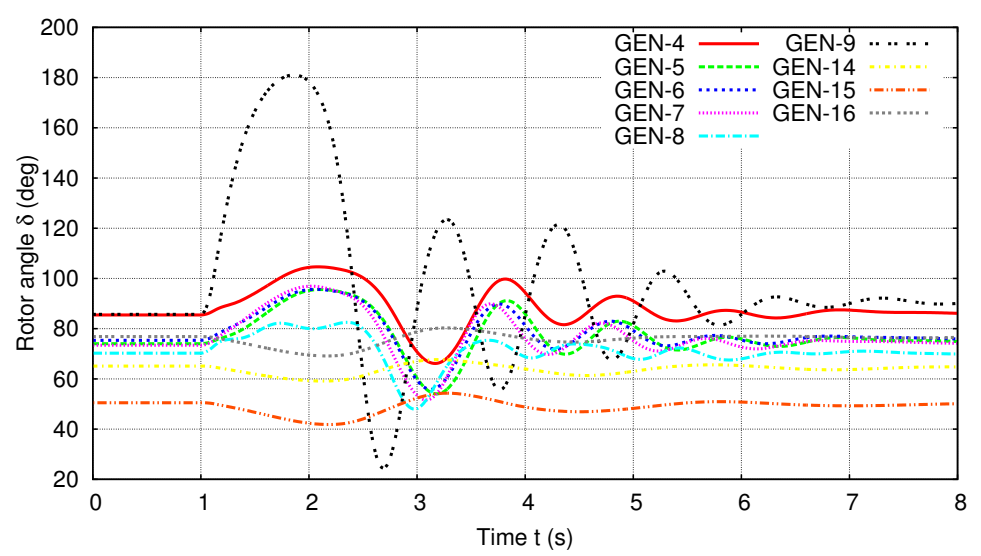

Figure 5: Evolution of rotor angles of a selection of generators (with respect to center of inertia)

buses which experience the lowest voltages (Bus 26, 27, 28 and 29), the three buses whose voltage magnitude are slightly increased (Bus 50, 51 and 52), and two buses little affected (Bus 18 and 25). As expected the voltage magnitudes drop dramatically in the fault-on period, and recover immediately after fault clearing. However, in the subsequent evolution, a voltage sag can be observed at certain buses. At some buses the voltage drops below the critical value of $0.7 \mathrm{pu}$, which is unacceptably low and long lasting.

\subsection{Identification of critical generator-load pairs}

In order to clearly identify which generators strongly impact load bus voltage magnitudes, the load voltage sensitivities described in the previous section are computed using Eq. (4) and data obtained from time-domain simulation, just after fault clearance. Figure 7 shows the resulting sensitivities. The bar graph shows for all load buses the expected change in voltage magnitude (in $\mathrm{pu} / \mathrm{rad}$ ) resulting from the increase of one individual generator rotor angle. The values clearly indicate that an angle increase can either depress or boost a voltage magnitude.

In the example scenario, GEN-9 experiences a large rotor angle excursions and is likely to cause the observed voltage sags. Indeed, Fig.7 7 indicates that GEN-9 mainly affects the voltage magnitudes at Buses 18 and $25-29$. Furthermore, from Fig. 5 it can be observed that GEN-14, GEN-15 and GEN-16 experience a slight decrease of their rotor angle. The sensitivities in Fig. 7 indicate that GEN-16 strongly affects the load buses with numbers in the range of $50-52$. In the following, a more detailed analysis of this 


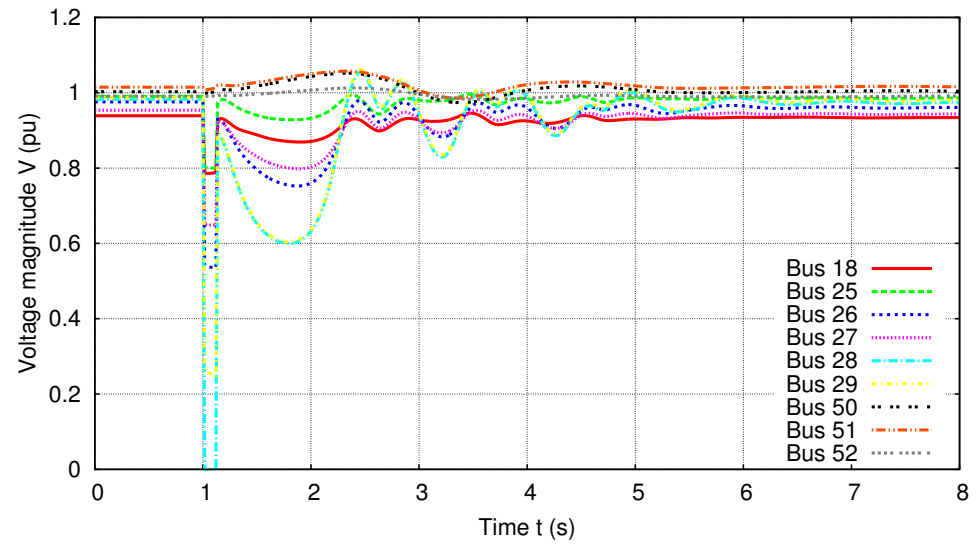

Figure 6: Evolution of voltage magnitudes at a selection of load buses

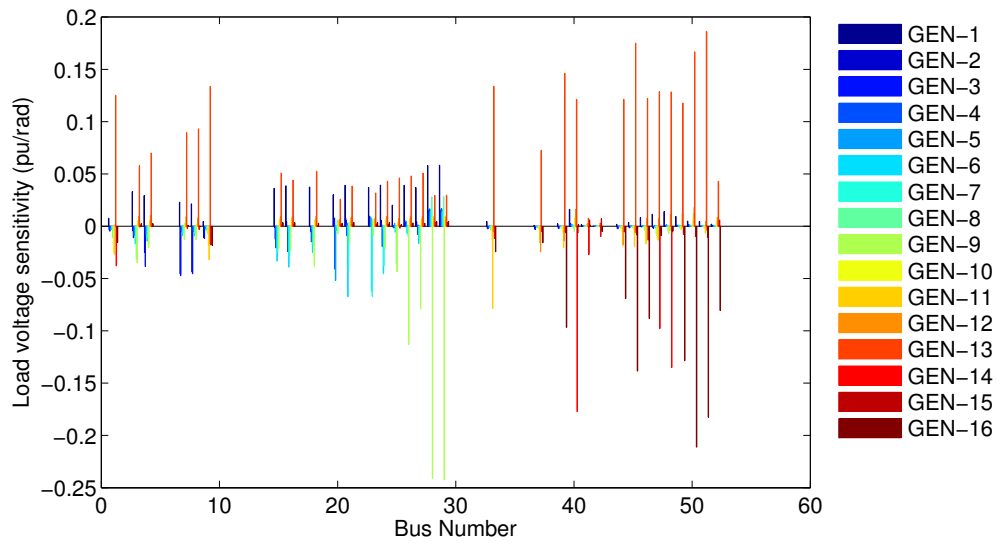

Figure 7: Load voltage sensitivities to a $1^{\circ}$ change in rotor angle of the individual generators 


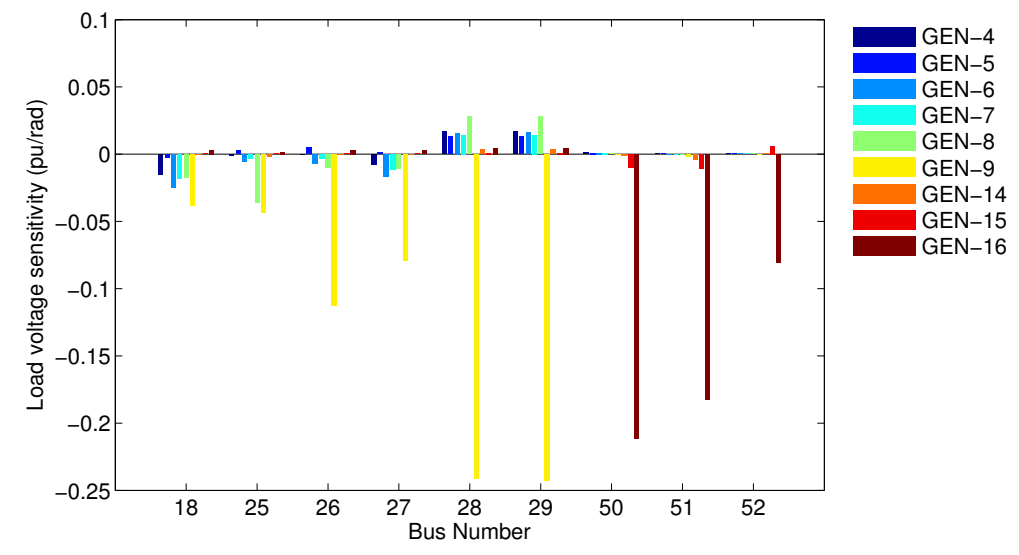

Figure 8: Load voltage sensitivities for a subset of loads and generators to a $1^{\circ}$ change in rotor angle

group is considered.

Figure 8 shows a selection of sensitivities displayed in the bar graph of Fig. 7, relative to above mentioned subset of generators and load buses. Dominant negative sensitivities indicate that increasing the corresponding rotor angles would decrease the voltage magnitude at the respective load buses, and vice versa. These high sensitivities combined with the generator rotor angle deviations can explain the voltage magnitude evolutions at the load buses shown in Fig. 6. The sensitivities in Fig. 8 suggest that the load buses where GEN-9 is dominant, will experience a voltage depression, which is the case for Buses 26, 27, 28 and 29. The voltage at Bus 18 and 25 are less affected, which is in good correlation with the lower sensitivities to the critical generator GEN-9. Furthermore, Fig. 8 suggests that the load buses where GEN-16 is dominant experience a slight increase of their voltage magnitudes corresponding to the negative sensitivity and the decreasing rotor angle of GEN-16. This is in good agreement with the voltage evolutions shown in Fig. 6

\subsection{Contribution of individual generators}

Another use of the load voltage sensitivities is to determine the contribution of each generator to an observed voltage sag at a particular bus, as described in Section 3.2, more precisely by Eq. (7). For the same test scenario, the contributions $\Delta V_{i, k}^{*}$ of each generator to the voltage sags are presented in Fig. 9 .

As for the load voltage sensitivities, the individual contributions of the 


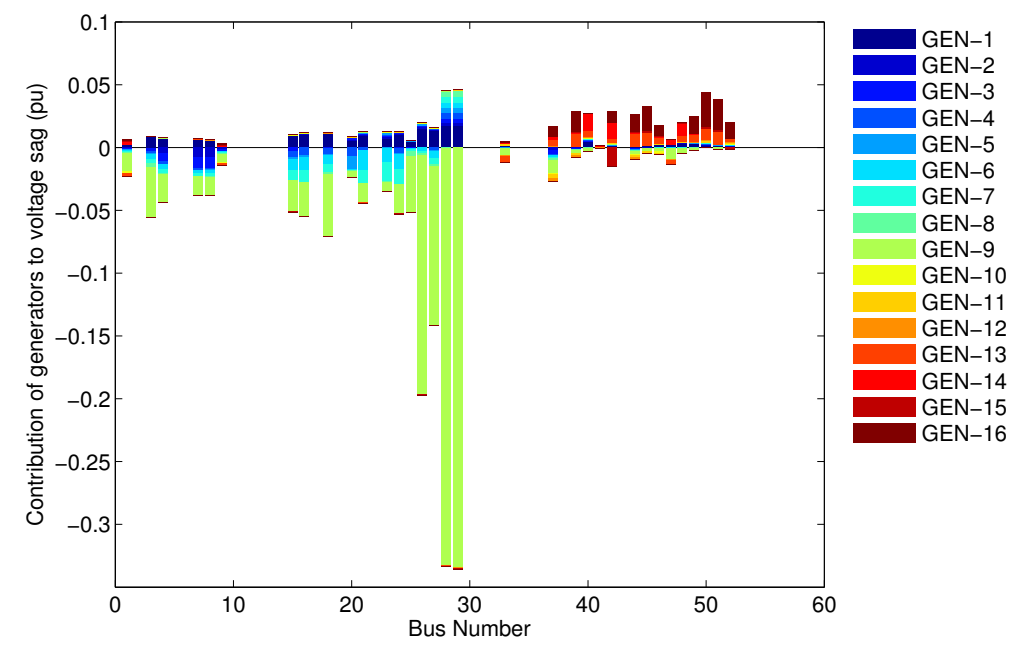

Figure 9: Contribution of individual generators to voltage depressions

generators show that each generator strongly impacts load buses in a certain vicinity and can have either a positive or a negative effect on the voltage magnitude. Moreover, it can be seen that, at an individual load bus, either a positive or a negative contribution can be dominant (see for instance Bus 28 , 29, 50 and 51), or the contributions of various generators compensate each other to a large extent (see for instance Bus 37).

\subsection{Application to generator re-dispatch}

The above individual generator contributions also point out effective locations for preventive control aimed at mitigating the voltage sags.

In the scenario under concern, GEN-9 has been identified as the major source of the voltage sags. Consequently, a reduction of its rotor angle excursion due to the fault could significantly improve the post-fault voltage evolutions. This can be achieved by reducing the critical generator's prefault active power and re-dispatching the power difference to appropriate non-critical generators.

The results of such a re-dispatch are presented next. The power output of GEN-9 was reduced by merely 50 MW (from 850 to $800 \mathrm{MW}$ ) while the power output of GEN-8 was increased to cover the difference. These two generators were chosen, because GEN-9 has a large contribution to the severe voltage sags at Buses 26 to 29, while the sensitivities shown in Fig. 8 suggest that a rotor angle increase of GEN-8 tends to increase the voltage magnitudes at the most critical Buses 28 and 29. Alternatively, any other 


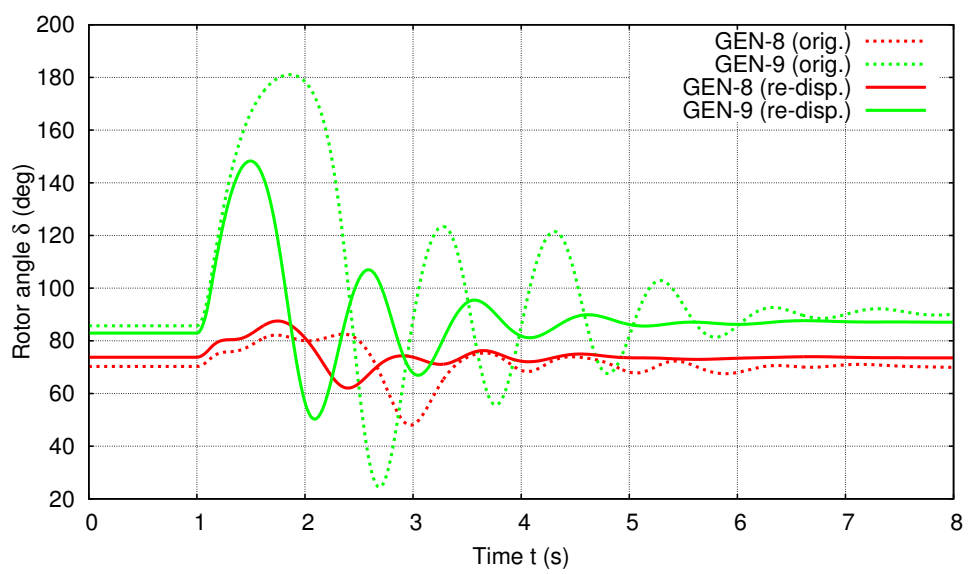

Figure 10: Rotor angle responses before and after power re-dispatch

generator with a negligible sensitivity could have been chosen to compensate the power reduction of the critical generator. In this test scenario the critical group of machines, which is the group that will loose synchronism in case of a fault cleared a little after the critical clearing time, consists of solely GEN-9.

Figure 10 shows a comparison of the rotor angle responses of GEN-8 and GEN-9, before and after the re-dispatch, respectively. It can be observed that, due to the active power re-dispatch, the large rotor angle excursion is significantly reduced for GEN-9. Furthermore, the duration of the first swing is almost halved. The beneficial effect on the voltage evolutions is evident in Fig. 11, showing the voltage evolutions at the load Buses 25-29. The depth of the voltage sag at each load bus is reduced dramatically and the duration of the swing is halved.

The individual generator contributions updated after the power re-dispatch are shown in Fig. 12, It can be observed that all the negative contributions are significantly lower in magnitude. At the same time, the load voltage sensitivities have been little affected by the small power re-dispatch. Consequently, the voltage improvement results essentially from the decreased rotor angle excursion of the critical generator.

The above example has demonstrated the usefulness of load voltage sensitivities and individual generator contributions to identify the source of voltage sags and appropriate locations for preventive control. It should be noted that, at this stage, those indexes do not provide information on the required amount of preventive control. 


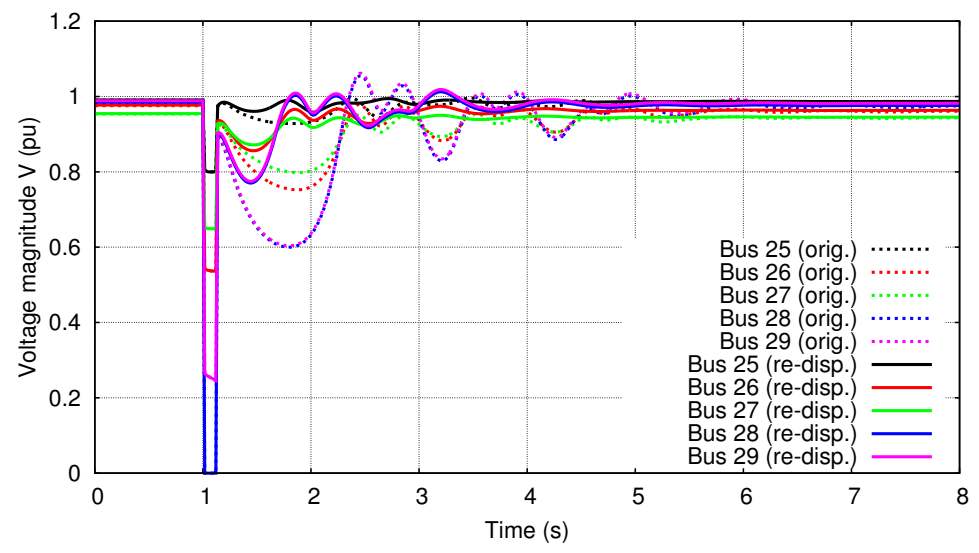

Figure 11: Voltage magnitude evolutions before and after power re-dispatch

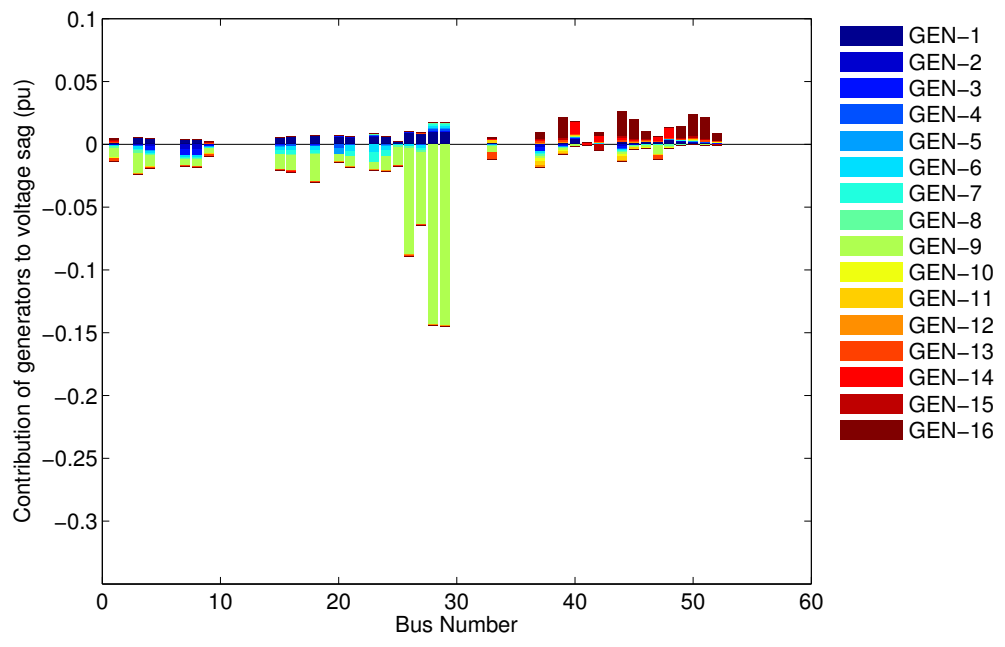

Figure 12: Contribution of individual generators to voltage depressions after power redispatch 


\section{Derivation of Generator Power Sensitivities}

\subsection{Motivation}

A depressed voltage, falling below a certain critical level, may trigger consecutive events such as load disconnection by internal protections, or possibly under-voltage load shedding in a system provided with this integrity protection scheme. The subsequent reduction of power consumption at some load buses may lower the maximum of the $P(\delta)$-curves of some generators. This leads to a reduction of the available deceleration area, which is detrimental for stability if those generators belong to the critical group, and beneficial if they belong to the non-critical one [11].

In order to investigate the effect of load variations on generator active powers, a second type of sensitivities is considered, based on the same model as in Section 2.1. The increase of load consumption is obtained from an extra load admittance $\Delta y$ added at the bus of concern, and the goal is to determine the effect on the generator active powers. It was found that these sensitivities provide the most accurate results when calculated directly after fault clearance, at time $t_{\mathrm{c}}^{+}$.

\subsection{Derivation of generator power sensitivities}

The first step consists in estimating the bus voltages after increasing the load admittance at the $k$-th bus by a small value $\Delta y$. To this purpose, let us assume that this additional admittance draws a current $\Delta \bar{I}_{k}$ from the network. The resulting change in bus voltages is given by:

$$
\Delta \overline{\boldsymbol{V}}=-\Delta \bar{I}_{k} \boldsymbol{Y}^{-1} \boldsymbol{e}_{k}
$$

where $\boldsymbol{e}_{k}$ is the unit vector with the $k$-th entry equal to one. The bus voltages after variation of the load admittance are given by:

$$
\overline{\boldsymbol{V}}=\overline{\boldsymbol{V}}^{\mathrm{o}}+\Delta \overline{\boldsymbol{V}}=\overline{\boldsymbol{V}}^{\mathrm{o}}-\Delta \bar{I}_{k} \boldsymbol{Y}^{-1} \boldsymbol{e}_{k}
$$

where the upper script ${ }^{\circ}$ denotes a value before the load change. This equation particularized to the $k$-th bus gives:

$$
\bar{V}_{k}=\bar{V}_{k}^{\mathrm{o}}-\Delta \bar{I}_{k}\left[\boldsymbol{Y}^{-1} \boldsymbol{e}_{k}\right]_{k}=\bar{V}_{k}^{\mathrm{o}}-\Delta \bar{I}_{k}\left[\boldsymbol{Y}^{-1}\right]_{k k}
$$

where $\left[\boldsymbol{Y}^{-1}\right]_{k k}$ is nothing but the Thévenin impedance seen from Bus $k$. Furthermore, the current $\Delta \bar{I}_{k}$ relates to the bus voltage $\bar{V}_{k}$ through:

$$
\Delta \bar{I}_{k}=\Delta y \bar{V}_{k}
$$


Combining Eq. 10 and Eq. (11) and solving for $\Delta \bar{I}_{k}$ yields:

$$
\Delta \bar{I}_{k}=\frac{\bar{V}_{k}^{\mathrm{o}}}{\left[\boldsymbol{Y}^{-1}\right]_{k k}+\frac{1}{\Delta y}}
$$

Replacing $\Delta \bar{I}_{k}$ by this expression in Eq. 9 provides the expression of bus voltages resulting from the addition of the admittance $\Delta y$ at the $k$-th bus:

$$
\overline{\boldsymbol{V}}=\overline{\boldsymbol{V}}^{\mathrm{o}}-\frac{\bar{V}_{k}^{\mathrm{o}}}{\left[\boldsymbol{Y}^{-1}\right]_{k k}+\frac{1}{\Delta y}} \boldsymbol{Y}^{-1} \boldsymbol{e}_{k}
$$

The second step consists in determining the variations of generator active powers that result from the change of voltages from $\overline{\boldsymbol{V}}^{o}$ to $\overline{\boldsymbol{V}}$. From the Thévenin equivalent of the $j$-th generator (Fig. $1(\mathrm{a})$, one easily derives the current before the load change:

$$
\bar{I}_{j}^{\mathrm{o}}=\left(\bar{E}^{\prime}-\bar{V}_{j}^{\mathrm{o}}\right) / j X_{j}^{\prime}
$$

and after the load change:

$$
\bar{I}_{j}=\left(\bar{E}^{\prime}-\bar{V}_{j}\right) / j X_{j}^{\prime}
$$

The variation of active power of the $j$-th generator is merely given by:

$$
\Delta P_{j}=\operatorname{Re}\left(\bar{V}_{j} \bar{I}_{j}^{*}-\bar{V}_{j}^{\mathrm{o}}\left(\bar{I}_{j}^{\mathrm{o}}\right)^{*}\right)
$$

where ${ }^{*}$ denotes the complex conjugate.

Equation (16) provides the sensitivities of the generator active powers to a change of the load admittance at the $k$-th bus. The main computational effort involves solving one sparse linear system from the LU factors of $\boldsymbol{Y}$ with the sparse independent term $\boldsymbol{e}_{k}$, which again is well suited for realtime application, e.g. determination of preventive controls. By repeating the computation for the various load buses of interest, a sensitivity matrix can be assembled column by column. As shown in the sequel, this matrix gives useful information on e.g. the effect of load tripping/shedding on generators during a transient voltage sag.

\section{Results for Generator Power Sensitivities}

\subsection{Investigation of the effect of load variation}

In the following an application of the power injection sensitivities is presented, where the sensitivities are used to estimate the effect of undervoltage load tripping. 


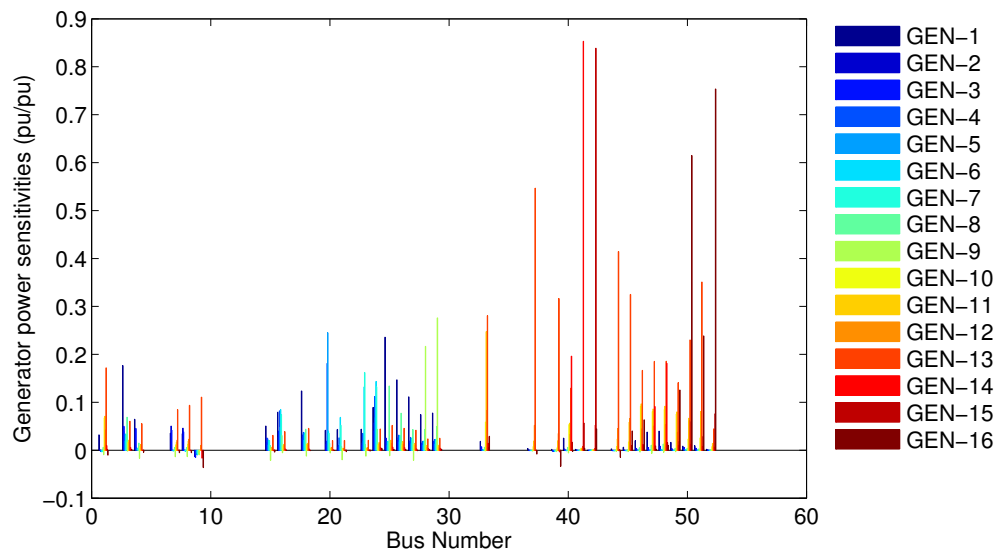

Figure 13: Generator power sensitivities to a 1 pu increase of the load admittance under constant power factor

Figure 13 shows the generator power sensitivities computed from Eq. (16) for the same scenario as in Section 4. The bar graph shows how much a $1 \mathrm{pu}$ increase of the load admittance under constant power factor affects the active powers of generators. It can be seen that increasing load power generally results in increasing generator active powers. However, in some rare cases, depending on the load power factor, the opposite effect can be observed. Moreover, it can be seen that there is a strong coupling between certain loads and certain generators, which was as well observed for the load voltage sensitivities.

Figure 14 shows a detail of the bar graph of Fig. 13. It can be seen that the power injection of GEN-9, which experiences a large rotor angle excursion and was found to have high impact on the voltages at Buses 28 and 29 , is as well sensitive to the load power consumed at these buses.

All in all, the relations between, on the one hand, voltage magnitudes at load buses and rotor angles and, on the other hand, active power of generators and load consumptions yield a more complete picture of the system response observed. Namely, the fault causes GEN-9 to have its rotor angles significantly increased. This, in turn, causes the voltage magnitudes at load Buses 28 and 29 to drop significantly, with the consequence that the voltage-sensitive load power is reduced. Eventually, the reduction of the power consumed by these loads further reduces the active powers of the generator, which results in a lower deceleration and, hence, a larger rotor angle excursion. 


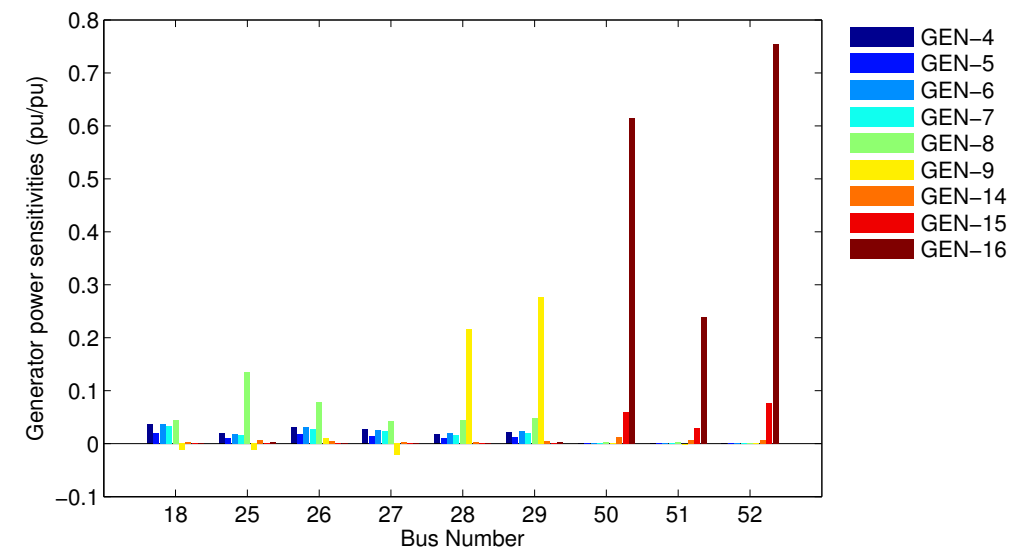

Figure 14: Excerpt of generator power sensitivities to a $1 \mathrm{pu}$ increase of the load admittance under constant power factor at prior selected buses

\subsection{Effect of load tripping/shedding}

Hereafter, the detrimental or beneficial effect of load tripping is discussed, based on the same scenario as before.

Figure 15 shows the voltage evolution at Bus 28 in three different cases. The red solid curve refers to the original scenario with a pronounced voltage sag. The green dashed curve is the same voltage evolution when $50 \mathrm{MW}$ of load are tripped at the Buses 28 and 29, under constant power factor, $100 \mathrm{~ms}$ after the voltage has dropped below the critical value of $0.7 \mathrm{pu}$. A loss of synchronism results, which can be explained from the sensitivities in Fig. 14. Indeed, reducing the load power at Buses 28 and 29 decreases the active power of GEN-9, which is the critical generator. The blue dotted curve shows the voltage evolution at Bus 28 when load is tripped at Bus 25. Stability is improved, the depth of the voltage sag is reduced and voltage recovery is faster. This is to be expected from the sensitivities in Fig. 14 which indicate that acting at Bus 25 little affects the critical generator GEN-9 but reduces the active power of GEN-8, which belongs to the non-critical group.

This observation could be at the heart of an intelligent load shedding scheme, detecting a voltage sag caused by rotor angle separation and selecting the loads to curtail in order to improve the system response. The case shown with the blue dotted curve in Fig. 15 was obtained by assuming a response-based scheme of this type, acting in several steps in order to apply a proper amount of corrective control. In this very simple example, a first block of $45 \mathrm{MW}$ of load is curtailed when the voltages stay below $0.7 \mathrm{pu}$ for more than $100 \mathrm{~ms}$, followed by one block of $45 \mathrm{MW}$ every $100 \mathrm{~ms}$ until all 


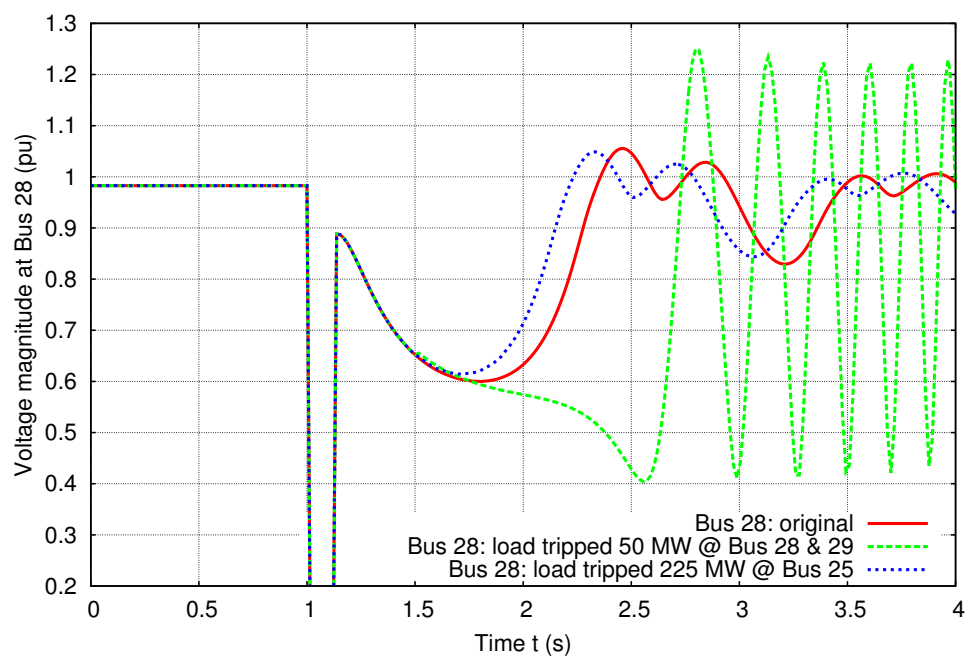

Figure 15: Effect of load shedding on the voltage magnitude at Bus 28

bus voltages recover above $0.7 \mathrm{pu}$. The power factor is kept constant. In the scenario of concern, this scheme leads to shedding a total of $225 \mathrm{MW}$. The effect on generator active powers is illustrated in Fig. 16, relative to the critical generator GEN-9. The red solid curve shows the evolution of its active power in the base case with no load shedding. The counterproductive load tripping scenario (50 MW shed at both Buses 28 and 29) is depicted by the green dashed curve. Load tripping takes place at $t=1.50 \mathrm{~s}$. The magnified plot in Fig. 16 shows that this slightly reduces the power injected by the generator. This decreases the deceleration power (i.e. the difference between the active and mechanical power) and eventually results in a loss of synchronism. In the stabilizing load tripping scenario, shown by the blue dotted curve (225 MW shed at Bus 25), the active power of the same generator is not reduced by the shedding. It is even higher than in the base case. This increase may be explained by a lower relative rotor angle between the critical and non-critical machines as well as a less pronounced voltage drop and, consequently, a higher power consumption.

\section{Conclusion}

In this paper, a method is proposed to assess transient voltage sags caused by rotor swings. This can occur when generators experience large relative rotor angle displacements after a fault. The system may be assessed transiently stable since no loss of synchronism takes place, but the voltage 


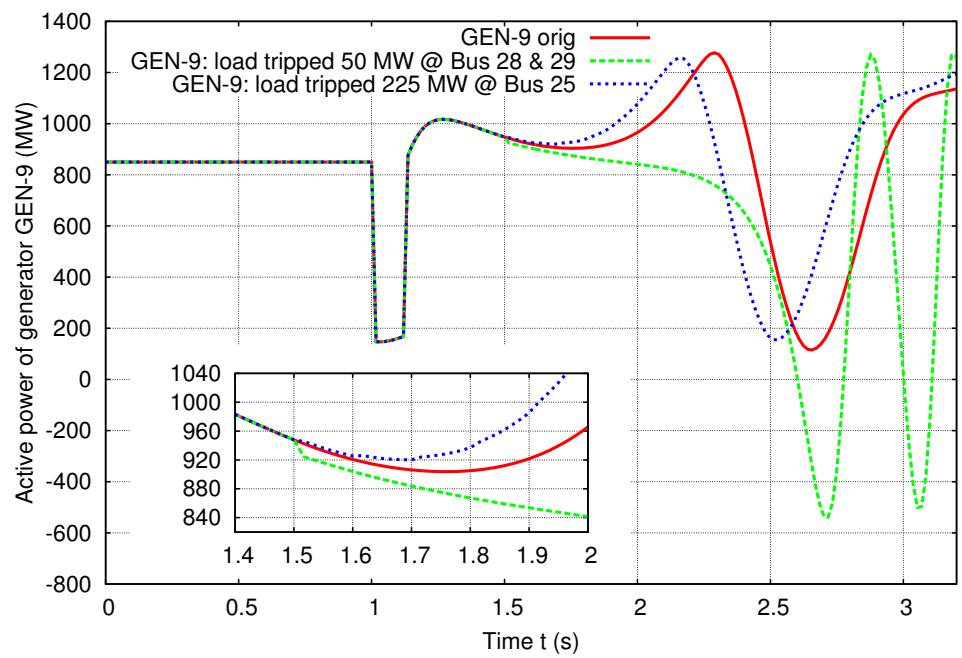

Figure 16: Effect of load shedding on active power of generator GEN-9

sag leads to critically low voltages and, hence, to an unacceptable system response.

The voltage sag assessment uses new sensitivities, derived from the wellknown linear algebraic network equations and aimed at complementing detailed time-domain simulations. To this purpose, generators are represented by the "classical" model adjusted to fit some points of the system evolution obtained with the full model. Under these assumptions, two sensitivities were developed.

The first sensitivity provides information on the impact of rotor angle changes on the voltage magnitude of load buses. This sensitivity can be used to detect critical generator-load pairs. Furthermore, it allows computing the contribution of a generator to an observed voltage sag, which gives valuable information to point out a location for preventive control. Presently, the sensitivity must be complemented with information on the amount of preventive measure. The second sensitivity reveals the impact of load consumption variation on the active power injected by generators. Simulations have shown that this sensitivity can be used to determine if under-voltage load tripping/shedding will improve or deteriorate transient stability and, thereby, identify critical locations for under-voltage load shedding.

The simulation results showed that although the sensitivities do not encompass all the existing controls, they provide useful information about the voltage sags caused by rotor swings. Moreover, since the sensitivities are obtained from a very fast computation, they could be used to determine 
preventive actions in real time. Assuming the availability of fast communication means, the sensitivities could also be used for emergency control. This extension of the work reported in this paper, however, requires a reliable early detection of voltage sags and proper tools to compute the amount and type of control.

\section{References}

\section{References}

[1] T. Weckesser, H. Jóhannsson, J. Østergaard, T. Van Cutsem, Sensitivity based Assessment of Transient Voltage Sags caused by Rotor Swings, in: Power Systems Computation Conference 18th, Vol. 1, IEEE, Wroclaw, Poland, 2014, pp. 1-7.

[2] M. H. Bollen, Understanding power quality problems, vol. 3 Edition, IEEE press, New York, 2000.

[3] A. A. Fouad, R. Sreedhara, Transient Voltage Dip Analysis using the Transient Energy Function Method, in: Proceedings of the TwentySecond Annual North American Power Symposium, IEEE Comput. Soc. Press, 1990, pp. 264-273. doi:10.1109/NAPS.1990.151379.

[4] F. Dominguez, A. S. Debs, J. Anasis, Transient voltage dip in power systems: computation and sensitivity analysis using the hybrid method, in: Decision and Control, 1992., Proceedings of the 31st IEEE Conference on, 1992, pp. 576-581 vol.1. doi:10.1109/CDC.1992.371665.

[5] Y. Xue, T. Xu, B. Liu, Y. Li, Quantitative assessments for transient voltage security, Proceedings of the 21st International Conference on Power Industry Computer Applications. Connecting Utilities. PICA 99. To the Millennium and Beyond (Cat. No.99CH36351) (1999) 101106doi:10.1109/PICA.1999.779391.

[6] A. Tiwari, V. Ajjarapu, Contingency assessment for voltage dip and short term voltage stability analysis, 2007 iREP Symposium - Bulk Power System Dynamics and Control - VII. Revitalizing Operational Reliability (2007) 1-8doi:10.1109/IREP.2007.4410569.

[7] D. Shoup, J. Paserba, C. Taylor, A survey of current practices for transient voltage dip/sag criteria related to power system stability, IEEE PES Power Systems Conference and Exposition, 2004. (2004) 14991506doi:10.1109/PSCE.2004.1397688. 
[8] P. Kundur, Power System Stability and Control, McGraw-Hill Inc., 1994.

[9] G. Rogers, Power System Oscillations, Springer, 2000.

[10] D. Molina, J. Liang, R. G. Harley, G. K. Venayagamoorthy, Virtual Generators: Simplified Online Power System Representations for WideArea Damping Control, in: IEEE Power and Energy Society General Meeting, 2012, pp. 1-8. doi:10.1109/PESGM.2012.6345608.

[11] M. Pavella, D. Ernst, D. Ruizvega, D. Ruiz-Vega, Transient Stability of Power Systems: A Unified Approach to Assessment and Control, Kluwer Academic Publishers, 2000. 